\title{
YAP inactivation in estrogen receptor alpha- positive hepatocellular carcinoma with less aggressive behavior
}

\author{
Youngsic Jeon ${ }^{1}$, Jeong Eun Yoo ${ }^{1}$, Hyungjin Rhee $\mathbb{C}^{2}$, Young-Joo Kim ${ }^{3}$, Gwang II Kim ${ }^{4}$, Taek Chung ${ }^{5}$, Sarah Yoon ${ }^{6,7}$,
} Boram Shin ${ }^{6,7}$, Hyun Goo Woo (10,7 and Young Nyun Park (1)

\begin{abstract}
The expression of estrogen receptor alpha (ERa, encoded by ESR1) has been shown to be associated with the prognostic outcomes of patients in various cancers; however, its prognostic and mechanistic significance in hepatocellular carcinoma (HCC) remain unclear. Here, we evaluated the expression of ERa and its association with clinicopathological features in 339 HCC patients. ERa was expressed in 9.4\% (32/339) of HCCs and was related to better overall survival (OS; hazard ratio $[H R]=0.11, p=0.009,95 \% \mathrm{C.I}=0.016-0.82)$ and disease-free survival $(\mathrm{DFS}, \mathrm{HR}=0.4$, $p=0.013,95 \%$ C.I. $=0.18-0.85)$. ERa expression was also associated with features related to more favorable prognosis, such as older age, lower serum alpha-fetoprotein level, and less microvascular invasion $(p<0.05)$. In addition, to obtain mechanistic insights into the role of ERa in HCC progression, we performed integrative transcriptome data analyses, which revealed that yes-associated protein (YAP) pathway was significantly suppressed in ESR1-expressing HCCs. By performing cell culture experiments, we validated that ERa expression enhanced YAP phosphorylation, attenuating its nuclear translocation, which in turn suppressed the downstream signaling pathways and cancer cell growth. In conclusion, we suggest that ERa expression is an indicator of more favorable prognosis in HCC and that this effect is mediated by inactivation of YAP signaling. Our results provide new clinical and pathobiological insights into ERa and YAP signaling in HCC.
\end{abstract}

\section{Introduction}

Hepatocellular carcinoma (HCC) is a highly malignant tumor with a dismal clinical prognosis. The incidence of HCCs is three times higher in males than in females ${ }^{1}$. Moreover, male patients showed worse overall survival (OS) than female patients ${ }^{2}$, implying that sex differences play an important role in HCC progression. Indeed, the sex hormone estrogen and the estrogen receptor (ER) have been found to be associated with the progression of

Correspondence: Hyun Goo Woo (hg@ajou.ac.kr) or Young

Nyun Park (young0608@yuhs.ac)

'Department of Pathology, Graduate School of Medical Science, Brain Korea 21 Project, Yonsei University College of Medicine, Seoul, Korea

${ }^{2}$ Department of Radiology, Yonsei University College of Medicine, Seoul, Korea

Full list of author information is available at the end of the article various cancers, including breast cancer, ovarian cancer, and $\mathrm{HCC}^{3,4}$.

The two major isoforms of ER are ER $\alpha$ (encoded by ESR1) and ER $\beta$ (encoded by ESR2), and ER $\alpha$ is the most abundant isoform in $\mathrm{HCC}^{5}$. Experimental studies have shown that ER $\alpha$ suppresses growth and inflammatory processes in diverse cancer types, including HCC, colorectal cancer, and gastric cancer ${ }^{6-8}$. Consistent with this observation, several studies have suggested that the expression of $E R \alpha$ is associated with better prognosis of HCC patients ${ }^{9,10}$. However, these studies were performed with a limited sample size, requiring further extended evaluation. Moreover, the precise mechanisms by which $\mathrm{ER} \alpha$ affects cancer progression are not yet clear.

In this study, to determine the prognostic significance of $\mathrm{ER} \alpha$ in HCC patients, we performed an extensive tissue

\section{(c) The Author(s) 2021}

(c) (i) Open Access This article is licensed under a Creative Commons Attribution 4.0 International License, which permits use, sharing, adaptation, distribution and reproduction cc) in any medium or format, as long as you give appropriate credit to the original author(s) and the source, provide a link to the Creative Commons license, and indicate if changes were made. The images or other third party material in this article are included in the article's Creative Commons license, unless indicated otherwise in a credit line to the material. If material is not included in the article's Creative Commons license and your intended use is not permitted by statutory regulation or exceeds the permitted use, you will need to obtain permission directly from the copyright holder. To view a copy of this license, visit http://creativecommons.org/licenses/by/4.0/. 
microarray (TMA) analysis of 339 HCC samples, which revealed that $\mathrm{ER} \alpha$ expression is an independent indicator of better prognostic outcomes in HCC patients. In addition, the results of our transcriptome data analyses suggested that inactivation of YAP may mediate the tumorsuppressive function of ER $\alpha$ in HCCs. YAP and transcriptional coactivator with PDZ-binding motif (TAZ) are the major downstream effectors of the Hippo pathway, which regulates tissue homeostasis, regeneration, and tumorigenesis $^{11-13}$. Activation of YAP or inhibition of upstream Hippo molecules such as the serine/threonineprotein kinases MST1/2 and large tumor suppressor kinase 1 (LATS1) can lead to nuclear translocation of YAP, which in turn promotes the tumorigenesis and progression of various cancers ${ }^{14,15}$. However, the effect of ER $\alpha$ expression on Hippo/YAP signaling has not yet been fully elucidated. In this study, we demonstrated that ER $\alpha$ expression can attenuate YAP signaling, resulting in suppression of HCC progression. Our results may provide new insights into the clinical and pathobiological significance of ER $\alpha$ and YAP signaling in HCC progression.

\section{Materials and Methods}

\section{Patients and specimens}

Patients who were diagnosed with HCC and underwent curative hepatic resection between May 2000 and February 2011 at Severance Hospital, Yonsei University Medical Center, were enrolled. A total of 339 HCC patients were included in the study, and patients with combined hepatocellular cholangiocarcinoma were excluded. None of the patients had received any preoperative treatment, such as transarterial chemoembolization, percutaneous ethanol injection, radiofrequency ablation, radiation, or systemic chemotherapy. Electronic medical records were reviewed to obtain clinical data, including age, sex, etiology, blood count, and the serum levels of aspartate transaminase, alanine transaminase, albumin, and the tumor marker alpha-fetoprotein (AFP). Histopathologic examination was performed on wholesection hematoxylin-eosin-stained slides prepared from representative formalin-fixed, paraffin-embedded tissue blocks. The following variables were evaluated: tumor size, Edmondson-Steiner grade, tumor multiplicity, tumor capsule formation, presence of microvascular invasion, and fatty change in the tumor.

Patients were routinely followed up by computed tomography or magnetic resonance imaging at intervals of 3-6 months. OS was defined as the time from surgery to death, and DFS was defined as the time from surgery to initial diagnosis of recurrence regardless of location. The mean follow-up time after surgery was $43.0 \pm 20.72$ months (0-146). The study was approved by the Institutional Review Board of Severance Hospital (IRB No. 4-2018-0748), and the requirement for informed consent was waived.

\section{Construction of tissue microarrays and immunohistochemistry}

TMAs of HCC specimens were constructed using two core biopsies of $2 \mathrm{~mm}$ in diameter, which were obtained from paraffin-embedded sections of tissue specimens and arranged into recipient TMA blocks using a trephine apparatus (Superbiochips Laboratories, Seoul, Korea). Immunohistochemistry was performed using antibodies against ER $\alpha$, EpCAM, K19, CD133, CD24, S100P, and YAP. Brown membranous and/or cytoplasmic staining was considered positive for EpCAM, K19, and CD133; cytoplasmic staining, for CD24; and nuclear staining, for $E R \alpha$ and S100P. Positivity for nuclear and cytoplasmic expression of YAP was concluded if more than $50 \%$ of the tumor cells were stained with strong intensity. The details on the antibodies and the experimental conditions are summarized in Supplementary Table S1.

\section{Transcriptome data analysis}

Public data from The Cancer Genome Atlas-Liver Hepatocellular Carcinoma (TCGA-LIHC) dataset were obtained by using the TCGAbiolinks package in R software $^{16}$, and the GSE87630, GSE4024, and GSE14520 datasets were obtained from the NCBI GEO database (https://www.ncbi.nlm.nih.gov/geo/). Integration of the datasets was performed by correcting batch effects using the "Combat" library in R software. Samples with normal tissues were excluded from the datasets.

Gene set analyses were performed using the gProfileR (0.7.0) package in $\mathrm{R}$ software and data from the KEGG (http://www.genome.jp/kegg/) and REAC (http://www. reactome.org/) databases. Coordinated gene regulation was identified using GSEA (https://www.gsea-msigdb.org/gsea, version 3.0). Gene sets from previous studies, including the gene sets for stemness/stem cell genes ${ }^{17}$ and the prognostic HCC classifiers of Hoshida's classification ${ }^{18}$ and Boyault's classification ${ }^{19}$. Prediction of the HCC groups by the prognostic classifiers was performed with the nearest template prediction (NTP) algorithm with a false discovery rate (FDR) $<0.05$ as the criterion for statistical significance as described previously $^{20}$. Detailed information on the datasets is provided in Supplementary Table S2.

The oncoactivity score was calculated based on the differential enrichment of oncogene activation tumor suppressor gene (TSG) repression as follows: Oncoactivity $=\mathrm{ES}_{\text {oncogene }}-\mathrm{ES}_{\mathrm{TSG}}$, where $\mathrm{ES}$ was the calculated enrichment score of the indicated genes. The lists of oncogenes $(n=674)^{21}$ and TSGs $(n=1088)^{22}$ were obtained from previous studies.

\section{Cell culture}

The human HCC cell lines HepG2 and Hep3B were purchased from the American Type Culture Collection (ATCC, Manassas, VA, USA) and grown in minimum 
essential medium (MEM, Gibco, Carlsbad, MD, USA), Dulbecco's modified Eagle's medium (DMEM, Gibco), and Roswell Park Memorial Institute 1640 medium (RPMI 1640, Gibco) supplemented with $10 \%$ fetal bovine serum (Gibco), $100 \mathrm{U} / \mathrm{mL}$ penicillin, and $100 \mu \mathrm{g} / \mathrm{mL}$ streptomycin at $37^{\circ} \mathrm{C}$ in a humidified atmosphere with $5 \% \mathrm{CO}_{2}$.

\section{Cloning and establishment of overexpression cell lines}

For the cloning of tagged ESR1 coding gene sequences into pCDH-CMV-EF1-puro, the ESR1 gene was amplified using total RNA extracted from normal liver tissue. PCR was performed using specific primers containing a $5^{\prime}$ extension and NotI (NEB, Ipswich, MA, USA) and XbaI (NEB) restriction sites with CloneAmp HiFi PCR Premix (Thermo Fisher Scientific, San Jose, CA, USA). Subsequently, the amplicons were digested with NotI and XbaI and cloned into the pCDH-CMV-EF1-puro vector using an In-Fusion ${ }^{\circledR}$ cloning system according to the manufacturer's recommendations. The details of the primer sequences and thermal cycling conditions are summarized in Supplementary Table S3.

HepG2 and Hep3B cells were transfected with pCDHCMV-EF1-puro containing the tagged ESR1 coding sequence along with the gag-pol and VSV-G plasmids (plasmids 14887 and 8454, respectively; Addgene, Cambridge, MA, USA) using Lipofectamine ${ }^{\circledR} 3000$ (Invitrogen, Carlsbad, CA, USA) according to the manufacturer's recommendations. After transfection, stable ESR1expressing cells were selected with $0.5-1.0 \mu \mathrm{g} / \mathrm{mL}$ puromycin (Sigma-Aldrich Co., St. Louis, MO, USA) for 4 weeks. To confirm the specificity and efficiency of ESR1 overexpression, we analyzed mRNA and protein expression using qRT-PCR and Western blotting.

\section{Immunofluorescence analysis}

Hep3B cells were seeded on microscope cover glasses in 12-well plates. After $24 \mathrm{~h}$, the cells were fixed with $4 \%$ paraformaldehyde for $30 \mathrm{~min}$. After permeabilization with PBS containing $0.2 \%$ Tween 20 for $30 \mathrm{~min}$ at $4{ }^{\circ} \mathrm{C}$, the cells were blocked with freshly prepared $2 \%$ bovine serum albumin (BSA) for $1 \mathrm{~h}$. The fixed cells were stained with an anti-YAP (1:400, sc-271134, Santa Cruz) antibody in $2 \%$ BSA for an hour at room temperature and then with Alexa Fluor ${ }^{\circledR} 488$-conjugated goat anti-mouse IgG as the secondary antibody for $1 \mathrm{~h}$ at room temperature. Finally, the cells were washed with cold PBS, and the cover glasses were mounted with DAKO $^{\circledR}$ Fluorescent Mounting Medium (DakoCytomation, Carpinteria, CA, USA). The cells were examined under a TCS SP5 confocal microscope system (Leica, Deerfield, IL, USA).

\section{Dual-luciferase reporter assay}

Cells were cotransfected with $100 \mathrm{ng}$ of each reporter construct and $0.25 \mathrm{ng}$ of the pNL1.1.TK vector
(Promega, Madison, WI, USA) per well in white-bottom 96-well plates (SPL Life Science, Pocheon, Korea) using $0.5 \mu \mathrm{l}$ of FuGENE ${ }^{\circledR}$ HD Transfection Reagent (Promega). To determine the effect of YAP/TEAD promoter activity, cells in each well were cotransfected with reporter constructs such as pGL3.0-basic (Promega) and 8xGITTC-luciferase (Addgene, plasmid \#34615, Cambridge, MA, USA) and the pNL1.1.TK vector. Forty-eight hours post transfection, luciferase activity was measured with a Nano-Glo ${ }^{\circledR}$ Dual Luciferase ${ }^{\circledR}$ Reporter Assay System (Promega) according to the manufacturer's recommendations. Relative firefly luciferase activity was normalized to NanoLuc ${ }^{\mathrm{Tm}}$ luciferase activity to adjust for variations in the transfection efficiency.

\section{Western blotting and quantitative real-time PCR analysis}

The primary antibodies used were rabbit anti-ER $\alpha$ (8644, Cell Signaling Technology [CST], Danvers, MA, USA), rabbit anti-phospho-YAP/S109 (13008, CST), rabbit anti-phospho-YAP/S127 (4911, CST), rabbit anti-YAP (14074, CST), rabbit anti-phospho-LATS/S1079 (8654, CST), rabbit anti-LATS1 (3477, CST), rabbit anti-phospho-MST1/2/T183/180 (3681, CST), rabbit anti-MST1 (3681, CST), rabbit anti-GAPDH (2118, CST), and rabbit anti-Histone H3 (cs-10809, Santa Cruz, California, CA, USA). Horseradish peroxidase-conjugated anti-rabbit IgG (7047, CST) and anti-mouse IgG (7076, CST) were used as secondary antibodies (for details, see Supplementary Table S1).

Quantitative real-time PCR was performed using iQTM SYBR Supermix (Bio-Rad, Hercules, CA, USA). The details of the primer sequences and thermal cycling conditions are summarized in Supplementary Table S3.

\section{siRNA-mediated knockdown experiments}

ESR1 siRNA (MISSION ${ }^{\circledR}$ select predesigned siRNAs; SASI_Hs01_00078594, Sigma-Aldrich Co.) and MISSION $^{\circledR}$ siRNA Universal Negative Control (SigmaAldrich Co.) were transfected into established ER $\alpha(-)$ and ER $\alpha(+)$ stable cells using Lipofectamine ${ }^{\circledR}$ RNAiMAX Transfection Reagent (Invitrogen) according to the manufacturer's recommendations. For the dual-luciferase reporter assay, $100 \mathrm{ng}$ of each construct, the pNL1.1.TK vector $(0.25 \mathrm{ng})$ and ESR1 siRNA $(50 \mathrm{nM})$ or siRNA Universal Negative Control $(50 \mathrm{nM})$ per well were cotransfected in 96-well white-bottom plates using Lipofectamine ${ }^{\circledR} 3000$ Reagent $(0.7 \mu \mathrm{l}$, Invitrogen) according to the manufacturer's recommendation.

\section{Statistical analysis}

Statistical analysis was conducted using SPSS (version 23.0.1; SPSS Inc., Chicago, IL, USA) or R software (version 3.4.0; Vienna, Austria). 


\section{Results}

ERa expression is associated with clinical and pathological features of HCC

ER $\alpha$ expression was evaluated using immunohistochemical staining of a TMA of samples from $339 \mathrm{HCC}$ patients. We observed that 9.4\% (32/339) of the HCCs expressed ER $\alpha$ (Fig. 1a). Clinicopathological features were evaluated according to the expression of ER $\alpha$, which revealed that compared to ER $\alpha(-) \mathrm{HCCs}, \mathrm{ER} \alpha(+) \mathrm{HCCs}$, were significantly associated with older age ( $>60$ years, $p<0.05$, Student's T-test), lower serum AFP levels (3.6 $\mathrm{IU} / \mathrm{mL}, p<0.001$, Student's T-test), a lower incidence of tumor microvascular invasion (38\%, 12/32, $p=0.029$, Fisher's exact test), and a higher incidence of fatty change in the tumor $(46.9 \%, 15 / 32, p=0.017$; Table 1). Kaplan-Meier survival analysis revealed that the patients with ER $\alpha(+) \mathrm{HCC}$ had better prognostic outcomes of OS (HR $=0.11, p=0.009$, Fisher's exact test) and DFS (HR = $0.40, p=0.013$, Fig. 1b). Univariate and multivariate Cox regression analyses also confirmed that ER $\alpha$ expression is an independent predictor of the prognostic outcomes of HCC patients $(p<0.05$, one-way Chi-square test Table 2$)$.

\section{Identification of the molecular traits associated with ESR1 expression in HCC}

To evaluate the molecular characteristics associated with ER $\alpha$ expression, we analyzed the HCC transcriptome data of the TCGA-LIHC dataset $(n=371)^{16}$. The HCC patients were stratified according to the expression level of ESR1 mRNA into the ESR1-high (ESR1-H, $n=185$ ) and ESR1-low (ESR1-L, $n=186$ ) groups, with the median value of the ESR1 level across the samples considered the cutoff value. Unsupervised clustering analysis using variably expressed genes (median absolute deviation [MAD] $>0.5, n=7094$ ) revealed that the transcriptome of the HCC patients was readily classifiable based on the ESR1 expression status. Specifically, 155 of 185 ESR1-H HCCs $(83.8 \%)$ and 148 of 186 ESR1-L HCCs (79.6\%) were clustered together (Supplementary Fig. S1), which may indicate that the molecular traits of ER $\alpha$ expression are well reflected in the HCC transcriptome. Next, we identified the differentially expressed genes (DEGs) between the ESR1-H and ESR1-L HCC groups as the ESR1 signatures "Sig ESR1-L" $(n=482)$ and "Sig ESR1-H" $(n=785)\left(p<10^{-6}\right.$, permutation $t$-test with fold change [FC] >0.5; Supplementary Table S4). Sig ESR1-H was enriched with metabolism-related genes, including CYP2A6 (FC=3.83), CYP3A4 $(\mathrm{FC}=3.78), C Y P 8 B 1(\mathrm{FC}=$ 3.38 ), and $C Y P 1 A 2$ (FC=2.33) (Fig. 2a and Supplementary Fig. S2), whereas Sig ESR1-L exhibited enrichment with stemness-related or tumor aggressiveness-related genes, such as $A F P(\mathrm{FC}=-2.70), C D 24(\mathrm{FC}=-2.27)$, S100P $(\mathrm{FC}=-2.01)$, SPP1 $(\mathrm{FC}=-1.89), \operatorname{EPCAM}(\mathrm{FC}=$ -1. 83), and KRT19 (FC=-1.01). Moreover, we found that compared to the ESR1-H group, the -ESR1-L group exhibited higher expression of HALLMARK oncogenic features such as "MYC_TARGETS_V1" $(n=200)$, "E2F_TARGET" $(n=200)$, "G2M_CHECKPOINT" $(n=$ 200), "MYC_TARGETS_V2" $(n=58)$, and "UNFOLDED_PROTEIN_RESPONSE” $(n=113)$ (Supplementary Fig. S3). In addition, we evaluated the expression of the previously known prognostic classifiers of HCC (i.e., a

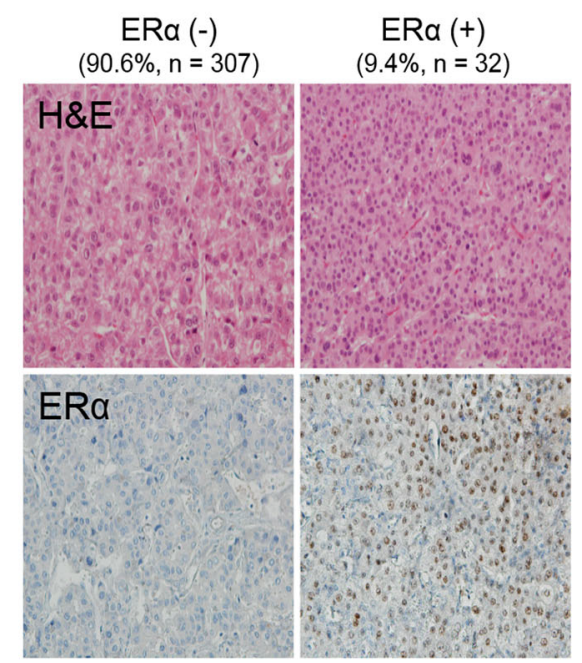

b
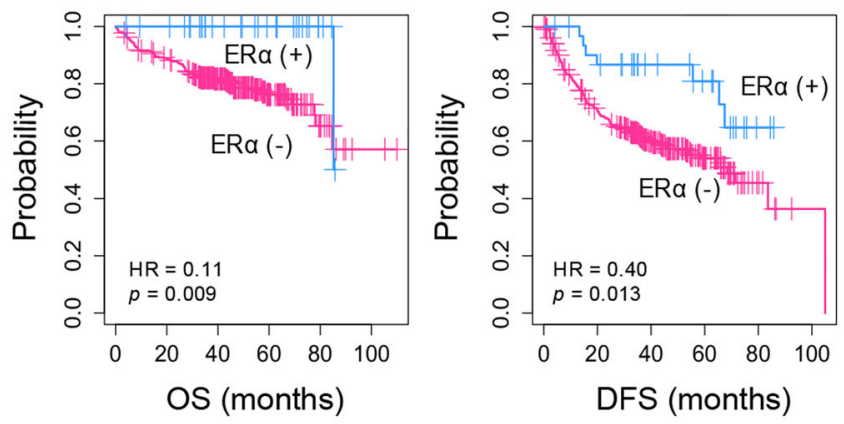

Fig. 1 ERa expression is associated with less aggressive clinical pathological features of HCC. a Histopathological images of ERa (-) and ERa (+) HCC tissue. ERa is expressed in the nuclei of tumor cells. b Kaplan-Meier curves of overall survival and disease-free survival for patients with ERa $(+)(n=32)$ and ERa $(-)$ HCC $(n=307)$. 
Table 1 Clinicopathological characteristics of patients with HCC according to the ERa protein expression level.

\begin{tabular}{|c|c|c|c|}
\hline Clinicopathological feature & $\begin{array}{l}\text { ERa protein-positive HCCs } \\
n=32(9.4 \%)\end{array}$ & $\begin{array}{l}\text { ERa protein-negative HCCs } \\
n=307(90.6 \%)\end{array}$ & $p$ value \\
\hline Age (years; median, IQR) & $60(51-68)$ & $55(48-63)$ & 0.015 \\
\hline Sex (male/female, \%) & $26(81 \%) / 6(19 \%)$ & $252(83 \%) / 53(17 \%)$ & 1 \\
\hline Etiology (HBV/HCV/Alcohol/Unknown, \%) & $25(78 \%) / 1(3 \%) / 1(3 \%) / 5(16 \%)$ & $252(82 \%) / 17(6 \%) / 14(5 \%) / 24(8 \%)$ & 0.462 \\
\hline Serum AST (IU/L; median, IQR) & $28(22-41)$ & $30(23-40)$ & 0.934 \\
\hline Serum ALT (IU/L; median, IQR) & $31(20-49)$ & $31(22-45)$ & 0.852 \\
\hline Serum albumin ( $\mathrm{g} / \mathrm{dL}$; median, IQR) & $4.3(4.1-4.6)$ & $4.4(4.1-4.7)$ & 0.61 \\
\hline Serum platelets (×1000/ML; median, IQR) & $174(119-201)$ & $162(133-210)$ & 0.343 \\
\hline Serum alpha-fetoprotein (IU/mL; median, IQR) ${ }^{a}$ & $3.6(2.5-27.1)$ & $24.9(4.6-279.5)$ & $<0.001$ \\
\hline Serum PIVKA-II (AU/mL; median, IQR) & $58.0(24.0-346.0)$ & $81.0(33.5-539.0)$ & 0.189 \\
\hline TNM stage (stage I/II/III, \%) & $17(53 \%) / 14(44 \%) / 1(3 \%)$ & $105(34 \%) / 186(61 \%) / 16(5 \%)$ & 0.104 \\
\hline \multicolumn{4}{|l|}{ HCC pathology } \\
\hline Differentiation (Edmonson-Steiner Grade I/II/III, \%) & $5(16 \%) / 22(69 \%) / 5(16 \%)$ & $21(7 \%) / 224(73 \%) / 62(20 \%)$ & 0.193 \\
\hline Diameter of the largest tumor ( $\mathrm{cm}$; median, IQR) & $2.7(1.8-4.3)$ & $3.2(2.2-4.5)$ & 0.782 \\
\hline Tumor multiplicity & $3(9 \%)$ & $41(13 \%)$ & 0.075 \\
\hline Tumor capsule formation (absent/partial/complete, \%) & $5(16 \%) / 14(44 \%) / 13(40 \%)$ & $54(18 \%) / 168(55 \%) / 85(28 \%)$ & 0.302 \\
\hline Microvascular invasion (\%) & $12(38 \%)$ & $182(59 \%)$ & 0.029 \\
\hline Tumor fatty change (absent/present, \%) & $17(53.1 \%) / 15(46.9 \%)$ & $223(73.1 \%) / 82(26.9 \%)$ & 0.017 \\
\hline \multicolumn{4}{|l|}{ Nontumor liver pathology } \\
\hline Chronic hepatitis or cirrhosis (\%) & $30(93.8 \%)$ & $294(95.8 \%)$ & 0.642 \\
\hline
\end{tabular}

IQR interquartile range, SD standard deviation.

aSerum alpha-fetoprotein was not available for one patient.

berum PIVKA-II was not available for 11 patients.

TCGA subtype ${ }^{23}$, Hoshida's classification ${ }^{18}$, Boyault's classification $^{19}$, and liver cancer stem cell features ${ }^{17}$ ) in the TCGA-LIHC dataset by applying the NTP algorithm (for details, see Materials and Methods). The ESR1-L group exhibited enrichment of the aggressive prognostic classifiers such as iCluster1 in TCGA subtype, S1 and S2 in Hoshida's classification, G1 and G3 in Boyault's classification, and UP in liver cancer stem cell features $(p<$ 0.001, chi-square test); in contrast, the ESR1-H group was enriched in the less aggressive prognostic classifiers, such as S3 in Hoshida's classification, G5 and G6 in Boyault's classification, and DOWN in liver cancer stem cell features $(p<0.001$, chi-square test, Fig. $2 \mathrm{~b})$. When we calculated the oncoactivity score for each sample based on the differentially upregulated expression of the oncogenes and counterbalanced suppression of the TSGs (i.e., $\mathrm{ES}_{\text {on- }}$ cogene-ESTSG) as described previously ${ }^{24}$ (for details, see Materials and Methods), we observed significantly higher oncoactivity scores in ESR1-L patients than in ESR1-H patients $\left(p<10^{-15}\right.$, Fig. 2 c). We also found that ESR1-L patients expressed stemness- and tumor aggressivenessrelated genes (CD24, S100P, EPCAM, and KRT19), which have been shown to play critical roles in the development and progression of tumors ${ }^{25,26}$. This finding was validated by immunohistochemical analysis of the 339 HCC specimens, demonstrating the differential expression of EpCAM, K19, CD133, CD24, and S100P between the ER $\alpha(+)$ and ER $\alpha(-)$ HCCs $(p<0.05$, Fisher's exact test; Fig. 2d). Correspondingly, Kaplan-Meier survival analysis revealed that the ESR1-H patients exhibited better OS ( $\left.\mathrm{HR}=0.53, p<10^{-3}\right)$ and DFS $\left(\mathrm{HR}=0.37, p<10^{-3}\right)$ than the ESR1-L patients (Fig. 2e). ESR1-H status was also significantly associated with older age $(>62$ years, $p<0.001)$ and a lower serum AFP level $(p<0.001)$ (Supplementary Table S5). Univariate and multivariate analyses confirmed that ESR1 expression was an independent prognostic predictor of the clinical outcomes of patients in TCGA-LIHC ( $p<0.05$, Supplementary Table S6). These results may strongly indicate that ER $\alpha$ (or ESR1) expression is a good indicator of better prognostic outcomes of HCC and is associated with alterations in the transcriptomic traits of HCC.

Next, we further validated the class predictability of the ESR1 signature by constructing a pooled dataset of HCC transcriptomes $(n=447)$, including data from GSE87630 
Table 2 Univariate and multivariate Cox regression analysis of overall survival of HCC patients.

\begin{tabular}{|c|c|c|c|c|c|c|c|}
\hline \multirow[t]{2}{*}{ Variable } & \multirow[b]{2}{*}{ No. of patients $(n=339)$} & \multicolumn{3}{|c|}{ Univariate analysis } & \multicolumn{3}{|c|}{ Multivariate analysis } \\
\hline & & HR & 95\% C.I. & $p$ value & $\mathrm{HR}$ & 95\% C.I. & $p$ value \\
\hline Age (years) & & 1.3 & $0.78-2.1$ & 0.33 & & & \\
\hline$\leq 60$ & 224 & & & & & & \\
\hline$>60$ & 115 & & & & & & \\
\hline Sex & & 1.4 & $0.74-2.7$ & 0.3 & & & \\
\hline Male & 277 & & & & & & \\
\hline Female & 62 & & & & & & \\
\hline Serum ALT (IU/L) & & 1.2 & $0.65-2$ & 0.62 & & & \\
\hline$\leq 50$ & 274 & & & & & & \\
\hline$>50$ & 65 & & & & & & \\
\hline Serum AST (IU/L) & & 1.8 & $0.95-3.2$ & 0.07 & & & \\
\hline$\leq 50$ & 299 & & & & & & \\
\hline$>50$ & 40 & & & & & & \\
\hline Alpha-fetoprotein & & 1.6 & $0.85-2.9$ & 0.15 & & & \\
\hline$\leq 1000$ & 294 & & & & & & \\
\hline$>1000$ & 44 & & & & & & \\
\hline Albumin & & 0.9 & $0.5-1.6$ & 0.32 & & & \\
\hline$\leq 4.5$ & 219 & & & & & & \\
\hline$>4.5$ & 120 & & & & & & \\
\hline Cirrhosis & & 1.4 & $0.84-2.2$ & 0.22 & & & \\
\hline Absent & 150 & & & & & & \\
\hline Present & 189 & & & & & & \\
\hline Tumor size & & 1.6 & $0.98-2.5$ & 0.62 & & & \\
\hline$<5 \mathrm{~cm}$ & 264 & & & & & & \\
\hline$\geq 5 \mathrm{~cm}$ & 75 & & & & & & \\
\hline Tumor multiplicity & & 1.5 & $0.76-2.9$ & 0.25 & & & \\
\hline Absent & 302 & & & & & & \\
\hline Present & 37 & & & & & & \\
\hline Microvascular invasion & & 1.7 & $1-2.7$ & 0.05 & 1.12 & $0.34-2.28$ & 0.81 \\
\hline Absent & 145 & & & & & & \\
\hline Present & 194 & & & & & & \\
\hline Differentiation & & 1.9 & $1.1-3.2$ & 0.027 & 1.76 & $0.64-4.79$ & 0.27 \\
\hline । & 122 & & & & & & \\
\hline$\|-\mid\|$ & 217 & & & & & & \\
\hline ERa protein expression & & 0.24 & $0.07-0.8$ & 0.031 & 0.25 & $0.08-0.83$ & 0.038 \\
\hline Negative & 307 & & & & & & \\
\hline Positive & 32 & & & & & & \\
\hline
\end{tabular}

HR hazard ratio, $\mathrm{Cl}$ confidence interval. 


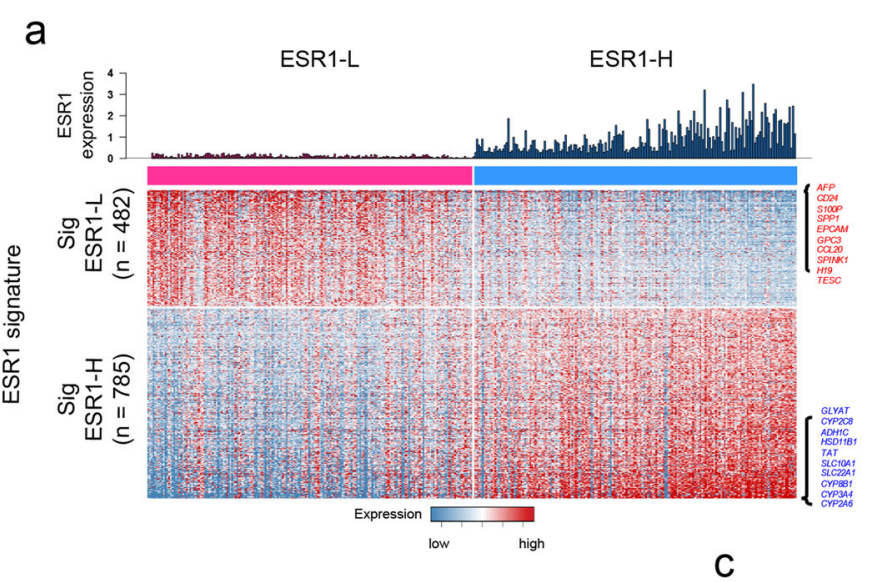

b
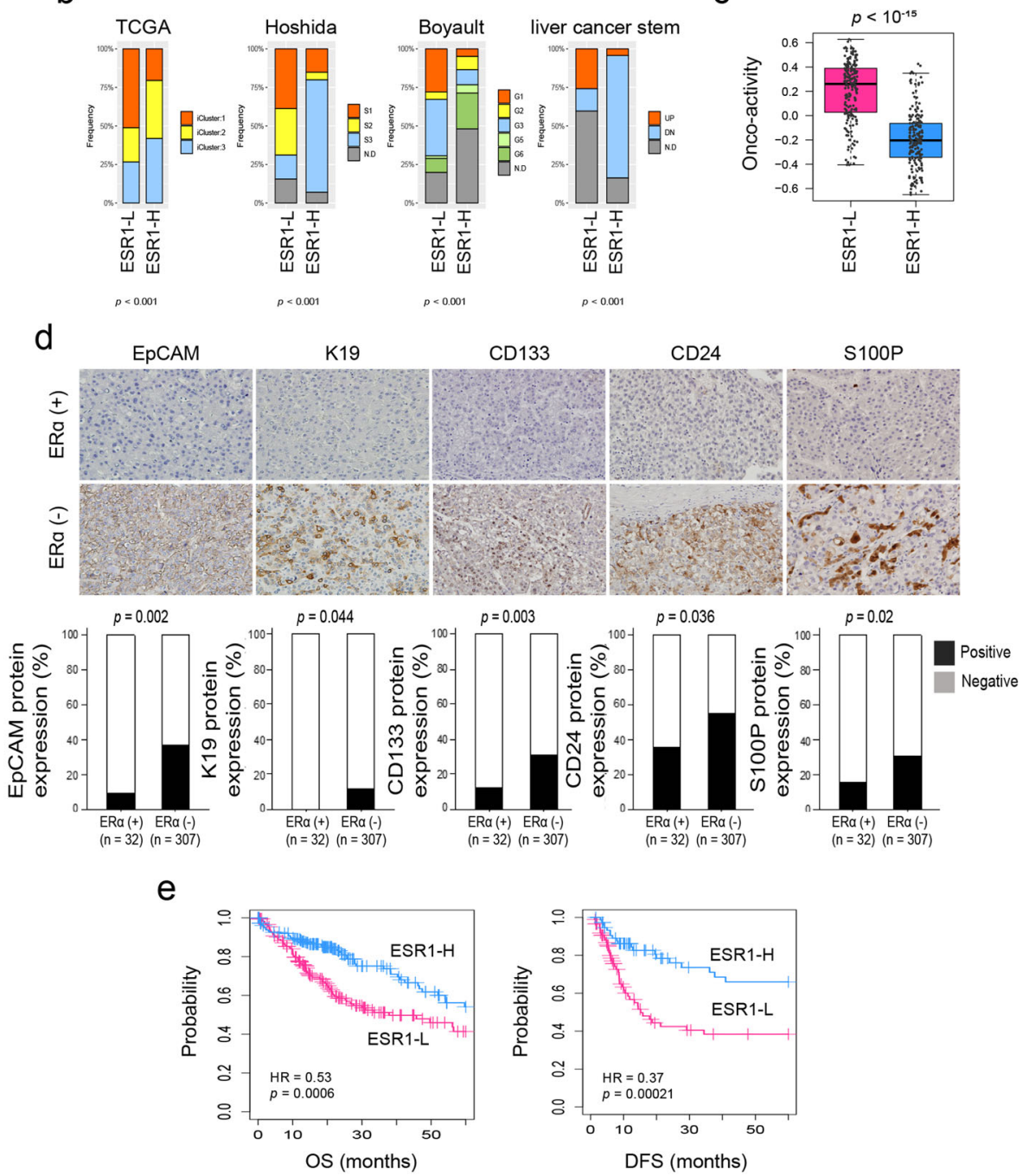

Fig. 2 Transcriptomic traits of HCC associated with the expression status of ESR1. a Heatmap showing the expression pattern of DEGS (Sig ESR1-L, $n=482$; Sig ESR1-H, $n=785$ ). The top ten DEGs are indicated on the right side. $\mathbf{b}$ Boxplots showing the frequencies of the subtypes predicted by the previous HCC classifiers (i.e., TCGA, Hoshida's, Boyault's, and liver cancer stem cell features). c Boxplot showing the oncoactivity scores among the HCC subtypes. d Images of immunohistochemical staining for EpCAM, K19, CD133, CD24, and S100P in the HCC TMA. Bar charts showing the differences in the expression of EpCAM, K19, CD133, CD24, and S100P between ERa (+) and ERa (-) HCCs. e Kaplan-Meier curves of OS and DFS for patients in the ESR1-H $(n=185)$ and ESR1-L $(n=186)$ groups in TCGA-LIHC. 


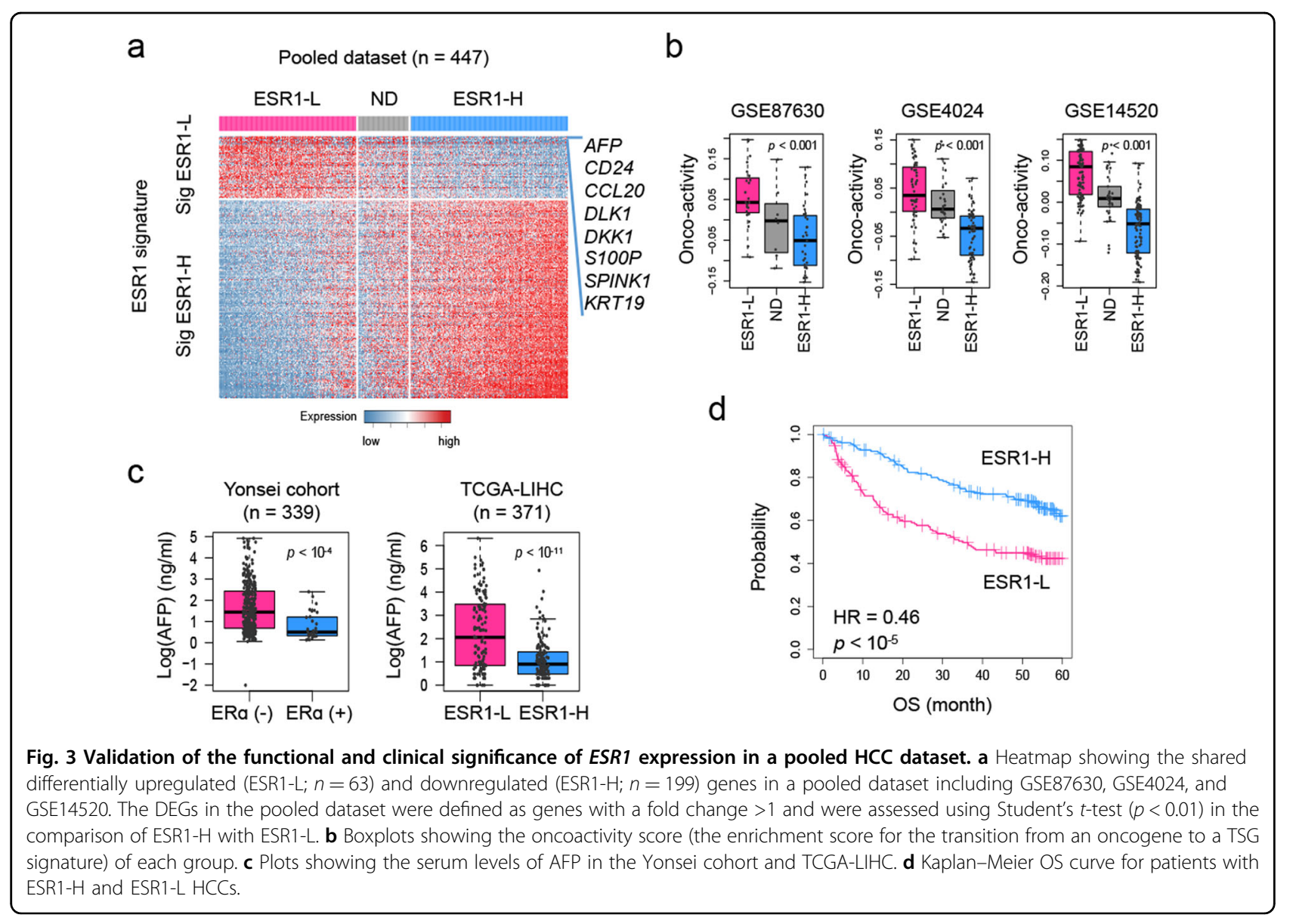

$(n=64)$, GSE4024 $(n=139)$, and GSE14520 $(n=244)$. By applying the NTP algorithm, we stratified the dataset into three groups: ESR1-H $(n=201)$, ESR1-L $(n=177)$, and N. D. $(n=69$, not determined, FDR $\geq .05$; Fig. $3 \mathrm{a})$. We demonstrated the higher oncoactivity scores in the ESR1L group compared to the ESR1-L group in each of the datasets $(p<0.001$, Fig. 3b; for details, see Material and Methods). In addition, we identified the DEGs between the ESR1-H and ESR1-L groups $(p<0.01$, permutation $t$ test and FC $>1.0$ ), revealing that $A F P$ expression had the highest $\mathrm{FC}$ between the groups $\left(\mathrm{FC}=3.34, p<10^{-12}\right)$. In support of this finding, we observed that HCC samples from patients with higher serum levels of AFP $(>300 \mathrm{ng} /$ $\mathrm{mL}$ ) were more frequently ER $\alpha(-)$ tumors than ER $\alpha(+)$ tumors in our data and were more frequently ESR1-L tumors than ESR1-H tumors in TCGA-LIHC data $(p<$ 0.001, chi-square test; Fig. 3c). Kaplan-Meier survival analysis also successfully demonstrated the prognostic significance of ESR1 expression in the pooled dataset ( $n=$ $378, \mathrm{HR}=0.46, p<10^{-5}$; Fig. 3d). Considering these results collectively, we suggest that ESR1 expression plays a pivotal role in transcriptomic alterations in association with the heterogeneous progression of HCC.

\section{ERa expression inhibits HCC progression by inactivating the YAP pathway}

We next sought to identify the underlying key downstream molecules that potentially drive the aggressive phenotype of ESR1-L. We constructed a genetic network of Sig ESR1-L by using the KEGG and REAC databases (for details, see Materials and Methods) and found that the gene set of cell cycle-related genes $(9.95 \%, 42 / 482)$ was the most significantly enriched (Fig. 4a and Supplementary Fig. S2). We found that among the cell cyclerelated genes, YAP-related target genes (i.e., $M Y B L 2$, CDC20, BIRC5, AURKB, TOP2A, and CENPF) and G2/Mrelated genes were more highly differentially expressed in ESR1-L than in ESR1-H (Fig. 4b). Gene set enrichment analysis also revealed enriched expression of "YAP1_UP"27 and "YAP_CONSERVED_SIG" ${ }^{28}$ genes in ESR1-L $(p<0.05$, Fig. $4 c)$. Indeed, YAP binding to B-MYB (encoded by the MYBL2 gene) is known to induce the expression of G2/M genes ${ }^{29}$. Moreover, we found that the promoter regions of the Sig ESR1-L genes frequently contained the predicted transcription factor (TF) binding sites for TEA domain family members (TEADs) (Fig. 4d), which are known to be activated by $\mathrm{YAP}^{12}$. Thus, it is 


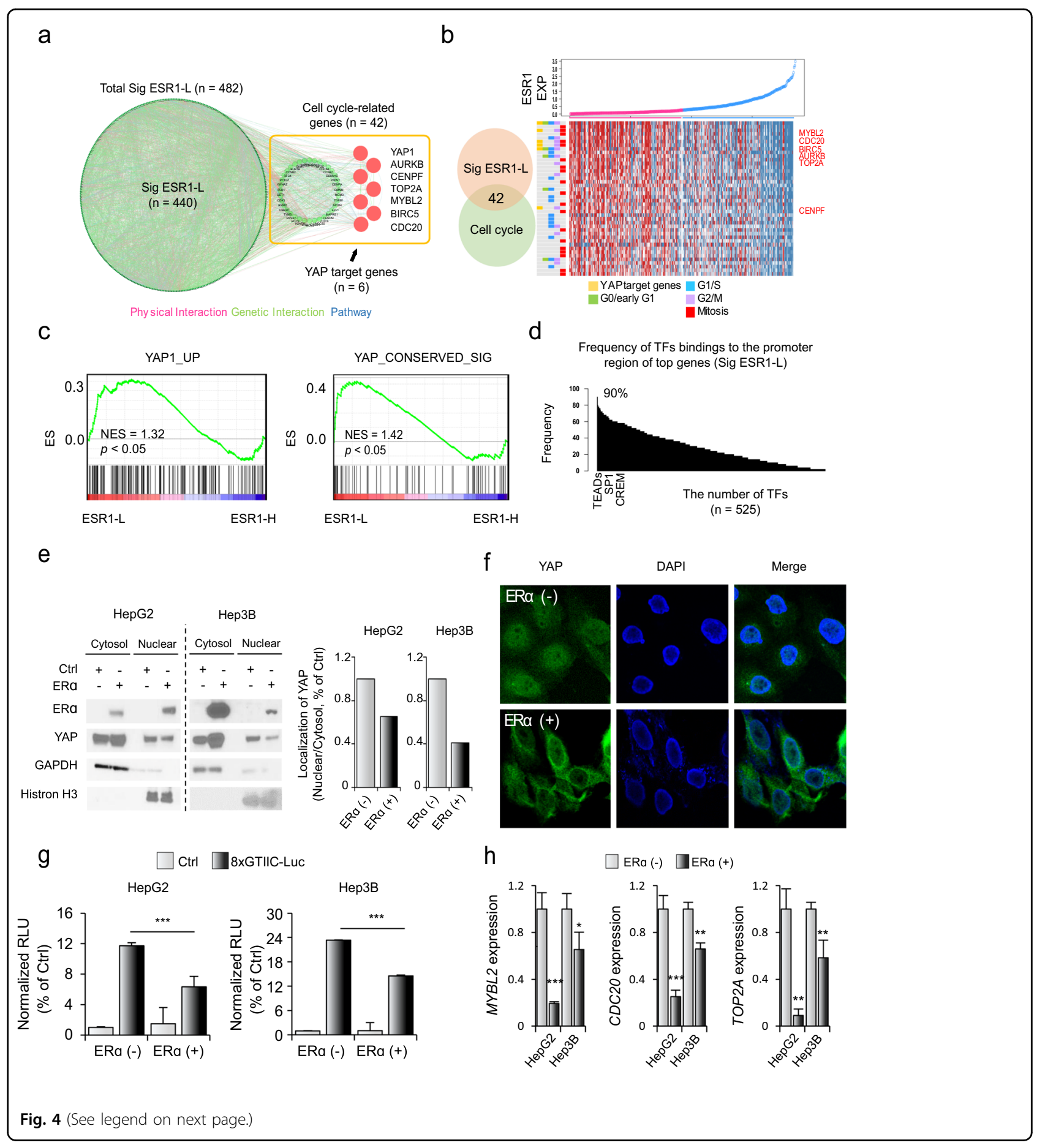

plausible that ESR1 expression may inactivate the YAP pathway, resulting in suppression of cell cycle-related genes and tumor progression.

To verify whether ESR1 expression can affect the YAP pathway, we established an ER $\alpha$ overexpression system in liver cancer cell lines (HepG2 and Hep3B) that have lower expression levels of ESR1 (Supplementary Fig. S4a). We demonstrated the tumor-suppressive effect of ER $\alpha(+)$ cells compared to $\mathrm{ER} \alpha(-)$ cells $(p<0.001$, Supplementary Fig. S4b). In addition, we evaluated whether ER $\alpha$ expression can regulate YAP activity by examining the cytosolic and nuclear levels of YAP using Western blot and immunofluorescence analyses. We demonstrated that compared to ER $\alpha(-)$ cells, ER $\alpha(+)$ cells significantly 
(see figure on previous page)

Fig. 4 YAP may mediate the aggressive phenotype of ESR1-L HCC. a A genetic network of Sig ESR1- $L$ genes was constructed showing physical interactions (pink), genetic interactions (green), and pathways (blue) by using GeneMANIA software in Cytoscape (version 3.4.1). Cell cycle-related genes and YAP target genes in Sig ESR1-L are indicated. b Plot showing the gradually increase in ESR1 expression (top). Heatmap showing the expression of YAP target genes and cell cycle-related genes in Sig ESR1-L (bottom). Cell cycle groups, such as G0/early G1-, G1/S-, G2/M-, and mitosisrelated groups, are indicated. c GSEA results showing the enrichment of YAP activation-related signatures (e.g., YAP1_UP and YAP_CONSERVED_SIG). d Bar plots showing the frequency of transcription factors binding to the promoters of the top-ranked genes in Sig ESR1-L $(n=50)$. This database was investigated using GeneHancer (https://www.genecards.org/). e The amount of YAP protein that translocated from the cytosol into the nucleus was assessed by Western blotting after nucleocytoplasmic fractionation. GAPDH and histone $\mathrm{H} 3$ were used as nuclear and cytoplasmic control markers, respectively (left). The amount of YAP protein that was translocated from the cytosol into the nucleus was assessed by Western blotting after nucleocytoplasmic fractionation using ImageJ (1.8.0_172) (right). f Confocal immunofluorescence of YAP and 4',6-diamidino-2-phenylindole (DAPI). DAPI (blue) and YAP (green) were detected by confocal microscopy as described in the Materials and Methods. $\mathbf{g}$ Dual-luciferase assays showed that the enhanced normalized relative luminescence unit (RLU) value of the 8xGTIIC construct was decreased in ERa-expressing HCC cell lines. The pGL3.0-basic vector was used as a control (Ctrl). Statistical significance is indicated (Ctrl vs. ERa; ${ }^{*} p<0.05,{ }^{* *} p<0.01$, and ${ }^{* * *} p<0.001$, Student's $t$-test). h Bar plots showing the expression of YAP target genes (e.g., MYBL2, CDC20, and TOP2A) in ERa (+) and ERa (-) liver cancer cell lines (HepG2 and Hep3B). The expression levels of each gene were normalized to the expression level of ACTB. The data were presented as the mean \pm SD values ${ }^{* * *} p<0.001$, Student's $t$-test).

suppressed the nuclear translocation of YAP (Fig. 4e, f). Furthermore, by performing YAP/TAZ reporter assays, we demonstrated that compared to ER $\alpha(-)$ cells, ER $\alpha(+)$ cells markedly suppressed YAP transactivation (all $p<$ 0.001 , Fig. 4g). Additionally, we observed that the expression of TEAD target genes (i.e., MYBL2, CDC20, and TOP2A) was suppressed in ER $\alpha(+)$ cells compared with ER $\alpha(-)$ cells (Fig. 4h). These results suggest that $E R \alpha$ expression leads to inactivation of YAP and suppression of its downstream target genes.

\section{ERa-induced YAP inactivation may be mediated by Hippo activation}

Next, to identify the mechanisms underlying YAP inactivation by ER $\alpha$, we examined the YAP phosphorylation status. Compared to ER $\alpha(-)$ cells, ER $\alpha(+)$ cells exhibited enhanced phosphorylation of YAP at Ser109 and Ser127 (Fig. 5a). As these sites were previously found to be phosphorylated by LATS1 and MAST1/2, core regulatory kinase proteins in the Hippo pathway ${ }^{30,31}$, we further evaluated whether Hippo signaling is also involved in ER $\alpha$-mediated YAP inactivation. We demonstrated that Hippo pathway genes (Reactome_signaling_by_hippo, $n=20$ ) were prominently expressed in the ESR1-H group compared to the ESR1-L group in the TCGA-LIHC dataset ( $p=0.048$, NES $=1.14$, Fig. $5 \mathrm{~b})$. In support of this finding, phosphorylation of the upstream Hippo signaling molecules LATS1 and MST1/2 was increased in ER $\alpha(+)$ cells compared with ER $\alpha(-)$ cells (Fig. 5c) ${ }^{12,32}$. These results suggest that ER $\alpha$-induced YAP inactivation is mediated at least in part through activation of Hippo signaling.

To further verify the effect of ER $\alpha$ on YAP, we performed small interfering RNA (siRNA)-mediated ESR1 knockdown experiments. Transfection of siRNAs targeting ESR1 suppressed ER $\alpha$-induced phosphorylation of YAP and MST1/2 (Fig. 5d). In addition, we evaluated
YAP/TAZ promoter activities by cotransfecting the YAP/ TAZ reporter construct with ESR1 siRNA, and the results revealed that ESR1 knockdown enhanced the suppressive effect of ER $\alpha$ on YAP/TAZ promoter activity (Fig. 5e). Furthermore, we validated our findings by immunohistochemical staining analysis of YAP in HCC tissues. ER $\alpha$ (-) HCC specimens showed increased nuclear expression of YAP $(34.9 \%, p=0.01)$ compared with that in ER $\alpha(+)$ HCC specimens (12.5\%), although the cytoplasmic expression levels of YAP were not different between the groups (Fig. 5f). Considering these results collectively, we suggest that ER $\alpha$ enhances the phosphorylation of Hippo/ YAP signaling molecules and attenuates downstream signaling in this pathway, which in turn results in suppression of HCC growth.

\section{Discussion}

In this study, we demonstrated that ER $\alpha$ expression is an independent predictor of better prognostic outcomes of HCC patients. In addition, by performing transcriptome data analyses, immunohistochemistry, and cell culture experiments, we demonstrated that the YAP pathway is involved in ER $\alpha$-mediated suppression of HCC growth.

Previously, numerous studies have shown that YAP/TAZ function as oncogenes in many cancers ${ }^{33}$. Activated YAP can sustain positive feedback for the expression of cell cycle-related genes to promote tumorigenesis ${ }^{34,35}$. Moreover, YAP has been shown to induce the expression of stemness-related genes, promoting an aggressive tumor phenotype $^{33}$. Indeed, YAP can reprogram nonstem tumor cells into cells with cancer stem cell attributes ${ }^{36}$. Correspondingly, the association of YAP with less favorable prognostic outcomes has been reported in $\mathrm{HCC}^{37,38}$. However, contradictory evidence for the tumor-suppressive functions of YAP/TAZ has also been reported ${ }^{39}$. A recent study showed that YAP/TAZ exert a tumor-promoting 


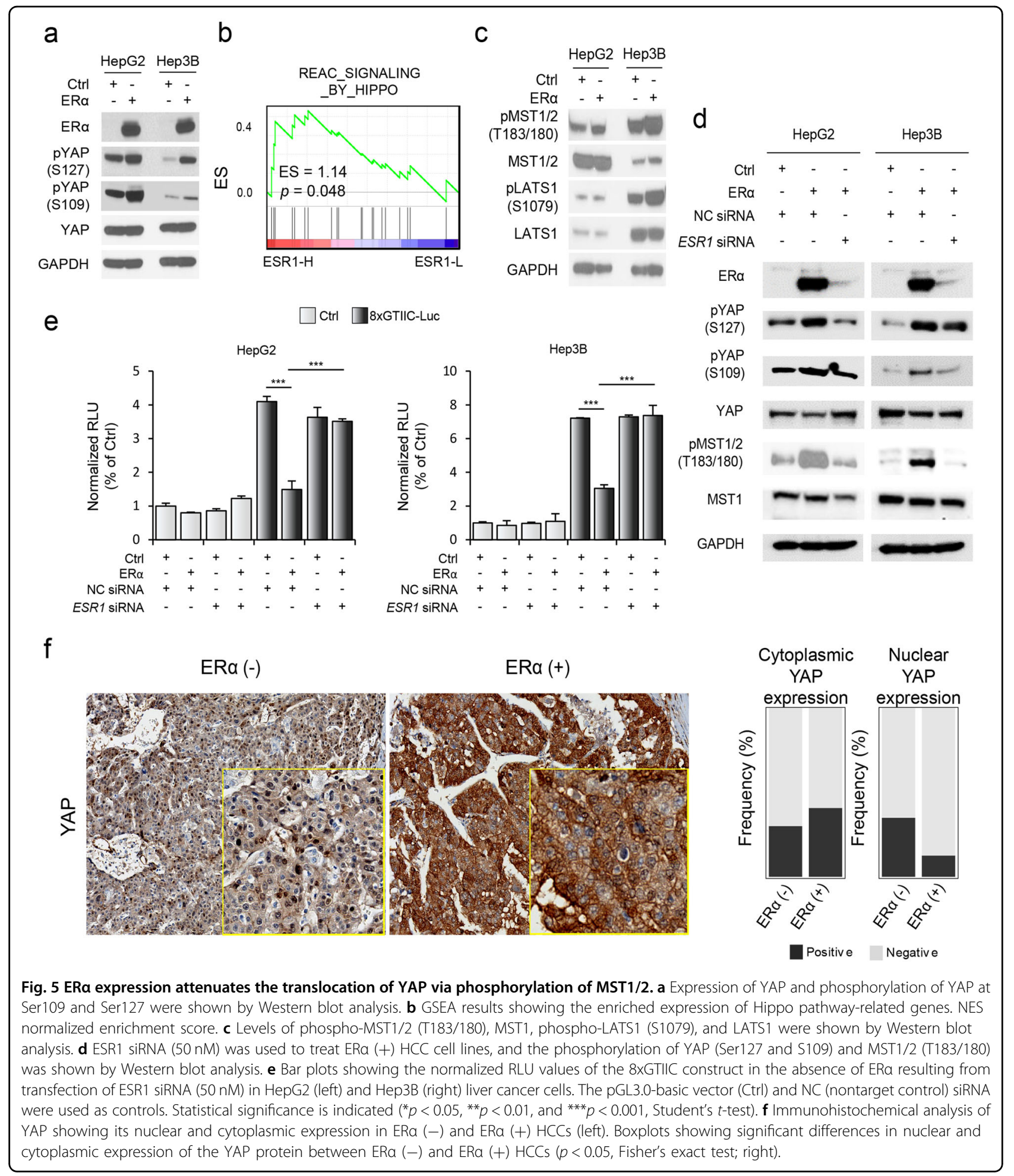

function but that activation of YAP/TAZ in peritumoral hepatocytes can suppress primary liver tumor progres$\operatorname{sion}^{40}$. This pattern may indicate that YAP/TAZ exhibit both tumor-suppressive and tumor-promoting activity depending on their expression in tissues.
We demonstrated that ER $\alpha$ inactivates YAP signaling, resulting in suppression of $\mathrm{HCC}$ growth. A recent study demonstrated that the YAP1 and TEAD4 proteins act as coregulators of ER $\alpha$ on enhancers ${ }^{41}$. Binding of YAP1/ TEAD4 to ER $\alpha$-bound enhancers is required for the 
induction of ER $\alpha$ target genes and tumor growth. However, the effect of ER $\alpha$ on the YAP pathway has not been clearly shown. We demonstrated that the expression of $E R \alpha$ triggers the phosphorylation of Hippo kinases (LATS1 and MST1) and YAP, which attenuates the nuclear translocation of YAP.

It should be noted that we did not evaluate how ER $\alpha$ phosphorylates Hippo/YAP proteins, requiring further extended studies in the near future. In addition, many of the multifaceted interactions of the Hippo/YAP pathways have been shown previously, including those that induce changes in mechanotransduction, inflammation, and oncogenic signaling ${ }^{33}$, which should be evaluated to understand more relevant mechanisms of ER $\alpha$ expression in HCC progression.

In conclusion, we suggest that the expression of ER $\alpha$ is an independent predictor of more favorable prognostic outcomes in HCC patients. Inactivation of YAP by ER $\alpha$ may contribute to the acquisition of a less aggressive phenotype by HCC tumors. Thus, targeting YAP could be a promising therapeutic strategy, especially for patients with ER $\alpha(-)$ HCC.

\section{Acknowledgements}

The authors thank Dong-Su Jang (Research Assistant, Department of Anatomy, Yonsei University College of Medicine, Seoul, Korea) for his excellent support with medical illustration. This research was supported by grants from the Myung-Sun Kim Memorial Foundation.

\section{Author details \\ ${ }^{1}$ Department of Pathology, Graduate School of Medical Science, Brain Korea 21 Project, Yonsei University College of Medicine, Seoul, Korea. ${ }^{2}$ Department of Radiology, Yonsei University College of Medicine, Seoul, Korea. ${ }^{3}$ Natural Products Research Center, Korea Institute of Science and Technology, Gangneung, Gangwon-do, Korea. ${ }^{4}$ Severance Biomedical Science Institute, Yonsei University College of Medicine, Seoul, Korea. ${ }^{5}$ Department of Biomedical Systems Informatics, Yonsei University College of Medicine, Seoul, Korea. ${ }^{6}$ Department of Physiology, Ajou University School of Medicine, Suwon, Korea. ${ }^{7}$ Department of Biomedical Science, Graduate School, Ajou University, Suwon, Korea}

\section{Author contributions}

Y.J. performed data analyses, experiments and data interpretation, and wrote the manuscript. J.E.Y., H.R., Y.-J.K., G.II.K., T.C. and B.S. participated in data acquisition, data analysis, and technical support. S.Y. acquired the stable cell lines. H.G.W. performed data analysis, wrote the manuscript, and supervised the study. Y.N.P. developed the study design, acquired and interpreted the data, wrote the manuscript, and supervised and funded the study.

\section{Conflict of interest}

The authors declare no competing interests.

\section{Publisher's note}

Springer Nature remains neutral with regard to jurisdictional claims in published maps and institutional affiliations.

Supplementary information The online version contains supplementary material available at https://doi.org/10.1038/s12276-021-00639-2.

Received: 8 December 2020 Revised: 17 April 2021 Accepted: 6 May 2021. Published online: 18 June 2021

\section{References}

1. Wu, E. M. et al. Gender differences in hepatocellular cancer: disparities in nonalcoholic fatty liver disease/steatohepatitis and liver transplantation. Hepatoma Res. https://doi.org/10.20517/2394-5079.2018.87 (2018).

2. Yang, D. et al. Impact of sex on the survival of patients with hepatocellular carcinoma: a surveillance, epidemiology, and end results analysis. Cancer 120, 3707-3716 (2014).

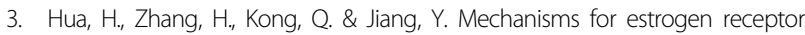
expression in human cancer. Exp. Hematol. Oncol. 7, 24 (2018).

4. Zhang, J. et al. Alternative splicing of estrogen receptor alpha in hepatocellular carcinoma. BMC Cancer 16, 926 (2016).

5. Iyer, J. K., Kalra, M., Kaul, A., Payton, M. E. \& Kaul, R. Estrogen receptor expression in chronic hepatitis $C$ and hepatocellular carcinoma pathogenesis. World J. Gastroenterol. 23, 6802-6816 (2017).

6. Ghebranious, N. \& Sell, S. Hepatitis B injury, male gender, aflatoxin, and p53 expression each contribute to hepatocarcinogenesis in transgenic mice. Hepatology 27, 383-391 (1998).

7. Barzi, A., Lenz, A. M., Labonte, M. J. \& Lenz, H. J. Molecular pathways: estrogen pathway in colorectal cancer. Clin. Cancer Res. 19, 5842-5848 (2013).

8. Chen, $\mathrm{C}$. et al. The roles of estrogen and estrogen receptors in gastrointestinal disease. Oncol. Lett. 18, 5673-5680 (2019).

9. Zhang, J. et al. Alternative splicing of estrogen receptor alpha in hepatocellular carcinoma. BMC Cancer 16, 926 (2016).

10. Villa, E. et al. Natural history of inoperable hepatocellular carcinoma: estrogen receptors' status in the tumor is the strongest prognostic factor for survival. Hepatology 32, 233-238 (2000).

11. Moroishi, T., Hansen, C. G. \& Guan, K. L. The emerging roles of YAP and TAZ in cancer. Nat. Rev. Cancer 15, 73-79 (2015).

12. Harvey, K. F., Zhang, X. \& Thomas, D. M. The Hippo pathway and human cancer. Nat. Rev. Cancer 13, 246-257 (2013).

13. Yu, F. X., Zhao, B. \& Guan, K. L. Hippo pathway in organ size control, tissue homeostasis, and cancer. Cell 163, 811-828 (2015).

14. Yimlamai, D. et al. Hippo pathway activity influences liver cell fate. Cell 157, 1324-1338 (2014).

15. Yin, F. et al. Spatial organization of Hippo signaling at the plasma membrane mediated by the tumor suppressor Merlin/NF2. Cell 154, 1342-1355 (2013).

16. Colaprico, A. et al. TCGAbiolinks: an R/bioconductor package for integrative analysis of TCGA data. Nucleic Acids Res. 44, e71 (2016).

17. Yamashita, T. et al. EpCAM-positive hepatocellular carcinoma cells are tumorinitiating cells with stem/progenitor cell features. Gastroenterology 136, 1012-1024 (2009)

18. Hoshida, Y. et al. Integrative transcriptome analysis reveals common molecular subclasses of human hepatocellular carcinoma. Cancer Res. 69, 7385-7392 (2009).

19. Boyault, S. et al. Transcriptome classification of HCC is related to gene alterations and to new therapeutic targets. Hepatology 45, 42-52 (2007).

20. Hoshida, Y. Nearest template prediction: a single-sample-based flexible class prediction with confidence assessment. PLoS ONE 5, e15543 (2010).

21. Liu, Y., Sun, J. \& Zhao, M. ONGene: a literature-based database for human oncogenes. J. Genet. Genomics 44, 119-121 (2017).

22. Zhao, M., Kim, P., Mitra, R., Zhao, J. \& Zhao, Z. TSGene 2.0: an updated literaturebased knowledgebase for tumor suppressor genes. Nucleic Acids Res. 44, D1023-1031 (2016)

23. Cancer Genome Atlas Research Network. Comprehensive and integrative genomic characterization of hepatocellular carcinoma. Cell 169, 1327-1341 e1323 (2017).

24. Jee, B. A. et al. Dynamics of genomic, epigenomic, and transcriptomic aberrations during stepwise hepatocarcinogenesis. Cancer Res. 79, 5500-5512 (2019).

25. Liu, Y. C., Yeh, C. T. \& Lin, K. H. Cancer stem cell functions in hepatocellular carcinoma and comprehensive therapeutic strategies. Cells https://doi.org/ 10.3390/cells9061331 (2020).

26. Hua, X. et al. Roles of $\mathrm{S} 100$ family members in drug resistance in tumors: status and prospects. Biomed. Pharmacother. 127, 110156 (2020).

27. Zhang, J., Smolen, G. A. \& Haber, D. A. Negative regulation of YAP by LATS1 underscores evolutionary conservation of the Drosophila Hippo pathway. Cancer Res. 68, 2789-2794 (2008).

28. Cordenonsi, M. et al. The Hippo transducer TAZ confers cancer stem cellrelated traits on breast cancer cells. Cell 147, 759-772 (2011).

29. Pattschull, G. et al. The Myb-MuvB complex is required for YAP-dependent transcription of mitotic genes. Cell Rep. 27, 3533-3546 e3537 (2019). 
30. Yu, F. X. et al. Regulation of the Hippo-YAP pathway by G-protein-coupled receptor signaling. Cell 150, 780-791 (2012).

31. Zhao, B., Li, L., Lei, Q. \& Guan, K. L. The Hippo-YAP pathway in organ size control and tumorigenesis: an updated version. Genes Dev. 24, 862-874 (2010)

32. Dey, A., Varelas, X. \& Guan, K. L. Targeting the Hippo pathway in cancer, fibrosis, wound healing and regenerative medicine. Nat. Rev. Drug Disco. 19, 480-494 (2020).

33. Zanconato, F., Cordenonsi, M. \& Piccolo, S. YAP/TAZ at the roots of cancer. Cancer Cell 29, 783-803 (2016).

34. Yang, S., Zhang, L., Chen, X., Chen, Y. \& Dong, J. Oncoprotein YAP regulates the spindle checkpoint activation in a mitotic phosphorylation-dependent manner through up-regulation of BubR1. J. Biol. Chem. 290, 6191-6202 (2015).

35. Kim, W. et al. Hippo signaling is intrinsically regulated during cell cycle progression by APC/C(Cdh1). Proc. Natl Acad. Sci. USA 116, 9423-9432 (2019).
36. Bartucci, M. et al. TAZ is required for metastatic activity and chemoresistance of breast cancer stem cells. Oncogene 34, 681-690 (2015).

37. Kim, G. J., Kim, H. \& Park, Y. N. Increased expression of Yes-associated protein 1 in hepatocellular carcinoma with stemness and combined hepatocellularcholangiocarcinoma. PLOS ONE 8, e75449 (2013).

38. $\mathrm{Xu}, \mathrm{M}$. Z. et al. Yes-associated protein is an independent prognostic marker in hepatocellular carcinoma. Cancer 115, 4576-4585 (2009).

39. Cottini, F. et al. Rescue of Hippo coactivator YAP1 triggers DNA damageinduced apoptosis in hematological cancers. Nat. Med. 20, 599-606 (2014).

40. Moya, I. M. et al. Peritumoral activation of the Hippo pathway effectors YAP and TAZ suppresses liver cancer in mice. Science 366, 1029-1034 (2019).

41. Zhu, C. et al. A non-canonical role of YAP/TEAD is required for activation of estrogen-regulated enhancers in breast cancer. Mol. Cell 75, 791-806.e798 (2019). 